\title{
Mathematical and Kinetic Modelling for Convective Hot Air Drying of Sweet Potatoes (Ipomoea batatas $L$ )
}

\author{
Inyang Uwem Ekwere*, Etuk Benjamin Reuben, Oboh Innocent Oseribho
}

Department of Chemical and Petroleum Engineering, University of Uyo, Uyo, Nigeria

Email address:

uweminyang@uniuyo.edu.ng (I. U. Ekwere)

${ }^{*}$ Corresponding author

\section{To cite this article:}

Inyang Uwem Ekwere, Etuk Benjamin Reuben, Oboh Innocent Oseribho. Mathematical and Kinetic Modelling for Convective Hot Air Drying of Sweet Potatoes (Ipomoea batatas L). Science Research. Vol. 7, No. 1, 2019, pp. 22-31. doi: 10.11648/j.ajche.20190701.13

Received: January 22, 2019; Accepted: March 8, 2019; Published: April 1, 2019

\begin{abstract}
The kinetic modelling of thin layer drying is performed with various agricultural products such as seeds, grains, fruits and some plant species with economic importance. Sweet Potato (Ipomoea batatas L) is a widely consumed, and its industrialization is on the increase. The thin layer drying kinetics of sweet potato were experimentally investigated in a convective hot air dryer. Comparison was made between the experimental and model predicted moisture ratio by nonlinear regression analysis. Furthermore, the effect of drying temperature and slice thickness on the selected model constants was evaluated. From the experimental data obtained, it was observed that moisture ratio of the potato sample was directly proportional to the mass of the sample while the time of drying was inversely proportional and these two parameters (mass and time) were used to develop a mathematical model for the thin layer drying of sweet potatoes using a convective hot air dryer. The mathematical model developed was validated. The experimental data fitted the developed mathematical model and gave the Sum of Squares (SSE) value of 0.0001296 and coefficient of determination $\left(R^{2}\right)$ as unity. The parameters obtained from the kinetic modelling of the selected models studied could be used in designing low cost dryers for optimum drying conditions.
\end{abstract}

Keywords: Sweet Potato, Moisture Ratio, Model Constant, Drying Kinetics, Thin Layer

\section{Introduction}

Drying operations had been applied for the reduction of moisture content in food materials for the prevention of microbial growth and deterioration, for shelf life elongation, to minimize packaging and improving storage for easy transportation. Although there are many ways in which drying can be achieved, the choice of a method depends on the material and the sanitary measures $[1,2]$. Fresh sweet potatoes are highly perishable due to their high moisture content and their availability is seasonal, so in some countries they are processed into various products by drying [3]. Therefore, it is necessary to predict the removal of moisture content of the sample (product) for preservation to avoid deterioration or spoilage [4]. Sweet Potato (Ipomoea batatas L.) is a tuberous crop of great importance in the world economy and feeding. It is widely consumed fresh, and its industrialization is on the rise, especially in the production of starch and chips [5]. Potatoes are also the fourth most important vegetable crop for human nutrition in the world [6]. Nigeria is among the major producers of potatoes, others are Uganda, China, Indonesia and Viet Nam [3]. Several investigators had proposed numerous mathematical models for the thin layer drying of many agricultural products and porous materials [7]. This process (thin drying method) is advantageous, because a full scale experimentation of different products and configurations of the drying system is time consuming and also costly [7]. Thin-layer equations are often used for a description of the drying kinetics for various types of porous materials. Thin-layer drying models that describe the drying phenomenon of biological materials fall mainly into three categories, namely, theoretical, semitheoretical and empirical. The first category takes into account only the internal resistance to moisture transfer, while the other two consider only the external resistance to moisture transfer between the product and the air $[2,8]$.

The aim of this study is to develop a mathematical model for predicting the thin layer drying conditions like mass and drying time using the hot air drying method and to fit 
experimental data to some selected kinetic models for the sweet potato under study.

\section{Materials and Methodology}

\subsection{Preparation of Samples}

The White Sweet Potatoes (Ipomea batatas L.) (Inyang UUH3729 (Uyo)) of the family of convolvulaceae were obtained from Itam market in Uyo, Akwa Ibom State and were identified in the department of Botany and Ecological Studies' herbarium. They were peeled and cut into a cylindrical shape of $30 \mathrm{~mm}$ in diameter using a locally fabricated peeler, after which they were sliced into various thickness of $2 \mathrm{~mm}, 4 \mathrm{~mm}, 6 \mathrm{~mm}$ and $8 \mathrm{~mm}$

\subsection{Drying Experiments}

Drying operation was carried out with a hot air Oven (model Wiseven 105). The potato slices were placed in a single layer on a wire mesh tray and placed inside the oven at different temperatures of $40^{\circ} \mathrm{C}, 50^{\circ} \mathrm{C}, 60^{\circ} \mathrm{C}$, and $70^{\circ} \mathrm{C}$. The initial weight of the sample used in the drying process was $200 \mathrm{~g}$ of the potatoes for the different thickness. The oven was switched on for 30 minutes [9], to simulate and keep the inside at required operating temperature, which gives steady state of operation before placing the samples inside the in oven every 30 minute interval. The wire mesh tray was removed from the oven and weighed using a digital weighing balance (Ohaus), [4, 10, 11]. The drying was continued until a constant weight was achieved under that drying condition. Aghbashlo et al. [6], [4, 12] among others asserted that moisture ratio could be determined from Equation (1):

$$
M R=\frac{M_{t}-M_{e}}{M_{o}-M_{e}}=\exp (-k t)
$$

where $M_{t}$ is the moisture content at any time $t$

$M_{o}$ is the initial moisture content of the sample

$M_{e}$ is the equilibrium moisture content

$k$ is the drying rate coefficient (reciprocal minutes)

$t$ is the time (minutes),

According to Aghbashlo et al. [6], the value of $M_{e}$ depends on the relative humidity and is negligible as the relative humidity is not constant throughout the entire drying process. Consequently, the moisture ratio was calculated according to the ASAE [13] standard as expressed in Equation (2):

$$
M R=\frac{M_{t}}{M_{o}}
$$

In order to determine the model(s) to predict the drying kinetics of sweet potato, the experimental moisture content data was used. The moisture content data for each drying process was estimated as a non-dimensional moisture ratio, due to the assumption of a uniform initial moisture content of all the samples [14, 15]. Furthermore, the best model(s) describing the thin layer drying characteristic of potato was chosen as the one with the highest $R^{2}$ value and the lowest SSE value [16, 17 and 18]. The statistical error used to minimize the difference between the experimental and models predicted data in order to achieve a good fit was the Sum of squares error (SSE). The goodness of fit of the models was established using coefficient of determination $\left(\mathrm{R}^{2}\right)$.

The experimental moisture ratio of the potatoes was fitted into thirteen (13) thin layer models widely utilized in describing the drying behaviour of agricultural products [12] as shown in Table 1.

\subsection{Moisture Content Determination}

The oven dry method described by ASAE [13] was used to determine the average initial moisture content of the samples. The samples were initially weighed using an electronic balance having a sensitivity of $0.001 \mathrm{~g}$ and placed in an air oven for 24 hours at $105^{\circ} \mathrm{C}$. After 24 hours, the samples were taken out of the oven and weighed to determine the final individual weight. The average values of the initial mass and final mass were used to calculate the moisture content expressed on a wet basis as shown in Equation 3. The process was repeated three times and the average was calculated.

$$
\text { Moisture content }(M C)=\frac{W_{i}-W_{f}}{W_{i}} \times 100
$$

Where $W_{\mathrm{i}}$ is the average initial weight of the sample and $W_{\mathrm{f}}$, is the average final weight of the sample.

\subsection{Model Development}

Amer et al. [19, 20] applied multiple linear regression analysis to establish mathematical equations for the drying kinetics which relate the drying process with the affecting factors. Literatures had established that the non - linear regression (or equation) technique could be used to develop a model which takes care of many variables $[21,22]$.

In developing the empirical model for drying of sweet potatoes, the following assumptions were made:

a. The moisture movement is only diffusion since most agricultural products use diffusion theory and this will be unidirectional.

b. The drying process is isothermal.

c. Shrinkage is neglected.

d. Materials to be dried is spherical but thin in size

e. Coefficient factor $\left(a_{o}\right)$, is independent of moisture concentration

For the development of the correlation in this study, the Moisture ratio (MR) depends inversely on time (as shown in Equation 4) and directly on mass (as shown in Equation 5). Based on this assertion, the relationships of MR with the drying parameters are presented in Equations (4) and (5):

$$
\text { (a) } M R \alpha \frac{1}{t}
$$




\section{(b) $M R \alpha M$}

Thus, the proposed correlation considers the proportionality of the MR to the different drying factors (mass and time); as presented in Equations 4 and 5.

In other words, the moisture ratio is expressed as a function of the mentioned parameters in Equation 6:

$$
M R=\mathrm{f}(\mathrm{t}, \mathrm{M})
$$

Equations 4 and 5 can be expanded in the basic form of nonlinear multivariable algebraic expression as given in Equation 7:

$$
M R=\alpha_{o}\left(M^{\alpha_{1}} \cdot t^{-\alpha_{2}}\right)
$$

Alternatively, we will have

$$
M R=\alpha_{o}\left[\frac{M^{\alpha_{1}}}{t^{\alpha_{2}}}\right]
$$

Applying the logarithmic transformation to linearize the Equation 8; we obtain

$$
\log (M R)=\log \left(\alpha_{o}\right)+\alpha_{1} \log (M)-\alpha_{2} \log (t)
$$

constants of the drying parameters.

The variables $\alpha_{o}$ through $\alpha_{2}$ could be determined iteratively using multivariable numerical optimization method. To determine these variables, existing literature indicates that, there are several approaches to performing this iterative estimation, namely, Gauss-Newton, the MarquardtLevenberg, the Nelder-Mead, the steepest descent, General Reduced Gradient (GRG) protocol [23-26].

To estimate the variables in the Equation 8 iteratively, these parameters (mass and time) were obtained experimentally. Then the unknown coefficient $\left(\alpha_{0}\right)$ and the exponential constants $\alpha_{1}$ and $\alpha_{2}$ were solved iteratively using GRG protocol in the Microsoft Excel SOLVER. To validate the correlation, its prediction was compared with the experimental data and some authors' correlation predictions. Also, the closeness of the correlation's predictions and experimental data were established using coefficient of determination $\left(\mathrm{R}^{2}\right)$.

\subsection{Kinetic Models for Thin Layer Drying}

Some kinetic models with parameters ranging from one to six were selected for fitting to the experimental data under study (see Table 1). The parameters were determined using the nonlinear regression method.

where $\alpha_{o}$ is a coefficient while $\alpha_{1}$ and $\alpha_{2}$ are exponential

Table 1. Mathematical models applied to the thin layer drying of various agricultural products.

\begin{tabular}{llll}
\hline S/N & MODEL NAME & MODEL EQUATION & REFERENCE \\
\hline 1. & Newton/Lewis & $M R=\exp (-k t)$ & {$[27]$} \\
2. & Page & $M R=\exp \left(-k t^{n}\right)$ & {$[28]$} \\
3. & Modified Page & $M R=\exp \left[(-k t)^{n}\right]$ & {$[29]$} \\
4. & Henderson and Pabis & $M R=a \exp (-k t)$ & {$[30]$} \\
5. & Logarithmic & $M R=a \exp (-k t)+c$ & {$[31]$} \\
6. & Two Term & $M R=a \exp \left(-k_{0} t\right)+b \exp \left(-k_{1} t\right)$ & {$[32]$} \\
7. & Two Term Exponential & $M R=a \exp (-k t)+(1-a) \exp (k a t)$ & {$[29]$} \\
8. & Singh et al & $M R=\exp (-k t)-a k t$ & {$[33]$} \\
9. & Aghabashlo Model & $M R=\exp -\left(\frac{k_{1} t}{1+k_{0} t}\right)$ & {$[34]$} \\
10. & Midilli et al & $M R=a \exp \left(-k t^{n}\right)+b t$ & {$[35]$} \\
11. & Hii et al & $M R=a \exp \left(-k_{1} t^{n}\right)+b \exp \left(-k_{2} t^{n}\right)$ & {$[36],[43]$} \\
12. & Demir et al & $M R=a \exp (-k t)^{n}+b$ & {$[37]$} \\
13. & Modified Midilli & $M R=\exp (-k t)+b t$ & {$[38]$} \\
\hline
\end{tabular}

$\mathrm{a}, \mathrm{b}, \mathrm{c}, \mathrm{k}, \mathrm{k}_{1}, \mathrm{k}_{2}$, and $\mathrm{n}$ are model constants

\subsection{Statistical Analysis}

The following statistical measures were used in determining the goodness of fit for the model $[2,12,39]$ :

(i) Chi Square $\left(\chi^{2}\right)=\sum_{i=1}^{n} \frac{\left(M R_{\exp }-M R_{p r e}\right)^{2}}{N-n}$ 
(ii) Residual Sum of Square $(R S S)=\sum_{i=1}^{n}\left(M R_{\exp }-M R_{\text {pre }}\right)^{2}$

(iii) Mean Bias Error $(M B E)=\frac{1}{N} \sum_{i=1}^{N}\left(M R_{\exp }-M R_{\text {pre }}\right)^{2}$

(iv) Root Mean Square Error $(R M S E)=\left[\sum_{i=1}^{n} \frac{1}{N}\left(M R_{\text {exp }}-M R_{p r e}\right)^{2}\right]^{\frac{1}{2}}$

(v) Coefficient of Determination $\left(R^{2}\right)=1-\left[\frac{\left(M R_{\exp }-M R_{p r e}\right)^{2}}{\left(M R_{\exp }-M R_{\text {preAVER }}\right)^{2}}\right]$

(vi) Sum of Square Error $(S S E)=\frac{1}{N} \sum_{i=1}^{N}\left(\frac{M R_{\text {exp }}-M R_{\text {pre }}}{N}\right)^{2}$

\section{Result and Discussion}

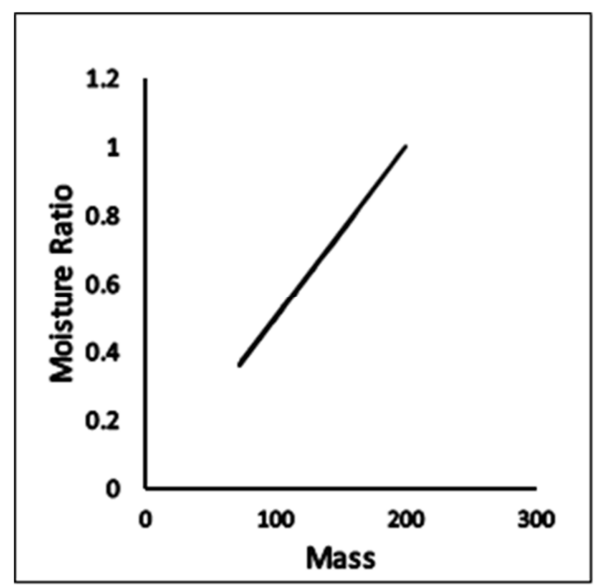

Figure 1. Moisture Ratio Versus Mass.

For the experiment, the experimental moisture content data were used to determine the moisture ratio. Thus, it was observed that Moisture Ratio (MR) depends directly on the mass (as shown in Figure 1) and inversely on time (as shown in Figure 2). In Figure 1, the moisture ratio increases as the mass increased while Figure 2 shows that the moisture ratio decreases as the time increased. Figure 1: Moisture Ratio Versus Mass Figure 2: Moisture Ratio Versus Time

The experimental data of sweet potato were fitted into thirteen thin layer drying models and these were compared with the developed model using the statistical indicators of $\mathrm{R}^{2}$ and SSE as listed in Table 2. The best model was the one with the highest $R^{2}$ value and the lowest SSE value.

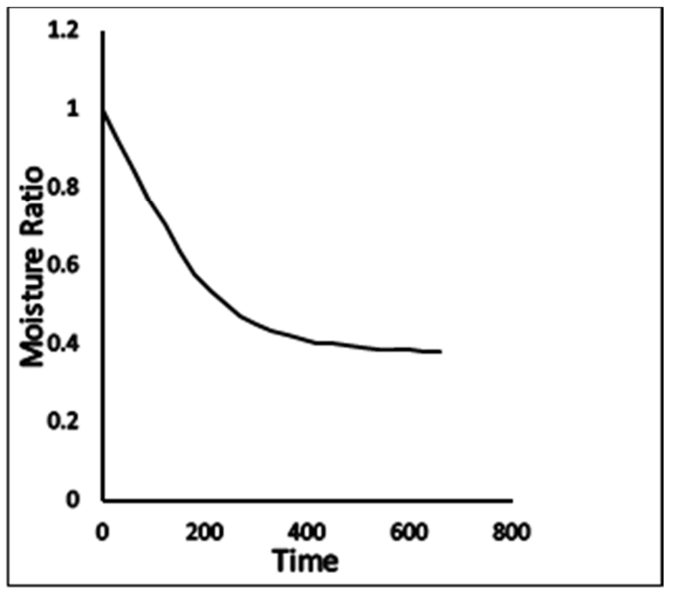

Figure 2. Moisture Ratio Versus Time.

Table 2. Statistical Comparison of selected models for drying of sweet potato.

\begin{tabular}{|c|c|c|c|c|c|c|c|c|c|}
\hline \multirow{3}{*}{ Model name } & \multirow{3}{*}{ Slice thickness (mm) } & \multicolumn{8}{|c|}{ Drying air temperature } \\
\hline & & \multicolumn{2}{|c|}{$40^{\circ} \mathrm{C}$} & \multicolumn{2}{|l|}{$50^{\circ} \mathrm{C}$} & \multicolumn{2}{|l|}{$60^{\circ} \mathrm{C}$} & \multicolumn{2}{|l|}{$7^{\circ} \mathrm{C}$} \\
\hline & & $\mathbf{R}^{2}$ & SSE & $\mathbf{R}^{2}$ & SSE & $\mathbf{R}^{2}$ & SSE & $\mathbf{R}^{2}$ & SSE \\
\hline \multirow[t]{4}{*}{ Lewis Model } & 2 & 0.7685 & 0.1307 & 0.1318 & 0.1752 & 0.9058 & 0.2051 & -0.4733 & 0.1572 \\
\hline & 4 & 0.7452 & 0.1445 & 0.4929 & 0.2408 & 0.9109 & 0.2167 & 0.1295 & 0.1944 \\
\hline & 6 & 0.9494 & 0.0445 & 0.3027 & 0.1118 & 0.7000 & 0.1299 & 0.1295 & 0.1944 \\
\hline & 8 & 0.9878 & 0.1752 & 0.8443 & 0.1003 & 0.6800 & 0.1735 & 0.5840 & 0.1108 \\
\hline \multirow[t]{3}{*}{ Page } & 2 & 0.9358 & 0.0361 & 0.8782 & 0.0243 & 0.8225 & 0.0293 & 0.7895 & 0.0224 \\
\hline & 6 & 0.9972 & 0.0136 & 0.9537 & 0.0312 & 0.9293 & 0.0306 & 0.8466 & 0.0342 \\
\hline & 8 & 0.9928 & 0.0064 & 0.9808 & 0.0123 & 0.9437 & 0.0305 & 0.9480 & 0.0230 \\
\hline \multirow[t]{4}{*}{ Modified page } & 2 & 0.1307 & 0.1307 & 0.1318 & 0.1752 & 0.2377 & 0.2051 & -0.4733 & 0.1572 \\
\hline & 4 & 0.1445 & 0.1445 & 0.4929 & 0.2408 & 0.7980 & 0.2167 & 0.1295 & 0.1944 \\
\hline & 6 & 0.0445 & 0.0445 & 0.8342 & 0.1118 & 0.9035 & 0.1299 & 0.1295 & 0.1944 \\
\hline & 8 & 0.0119 & 0.0119 & 0.8443 & 0.1003 & 0.9606 & 0.1735 & 0.7493 & 0.1108 \\
\hline
\end{tabular}




\begin{tabular}{|c|c|c|c|c|c|c|c|c|c|}
\hline \multirow{3}{*}{ Model name } & \multirow{3}{*}{ Slice thickness $(\mathbf{m m})$} & \multicolumn{8}{|c|}{ Drying air temperature } \\
\hline & & \multicolumn{2}{|c|}{$40^{\circ} \mathrm{C}$} & \multicolumn{2}{|l|}{$\mathbf{5 0}^{\circ} \mathrm{C}$} & \multicolumn{2}{|l|}{$60^{\circ} \mathrm{C}$} & \multicolumn{2}{|l|}{$70^{\circ} \mathrm{C}$} \\
\hline & & $\mathbf{R}^{2}$ & SSE & $\mathbf{R}^{2}$ & SSE & $\mathbf{R}^{2}$ & SSE & $\mathbf{R}^{2}$ & SSE \\
\hline \multirow[t]{4}{*}{ Two Term } & 2 & 0.9981 & 0.0019 & 0.9991 & 0.0002 & 0.9950 & 0.0008 & 0.9998 & 0.0000 \\
\hline & 4 & 0.9950 & 0.0028 & 0.9961 & 0.0018 & 0.9990 & 0.0005 & 0.9974 & 0.0006 \\
\hline & 6 & 0.9979 & 0.0007 & 0.9976 & 0.0016 & 0.9347 & 0.0650 & 0.9974 & 0.0006 \\
\hline & 8 & 0.9885 & 0.0103 & 0.9999 & 0.0000 & 0.9996 & 0.0002 & 0.9988 & 0.0005 \\
\hline \multirow[t]{4}{*}{ Two Term Exponential } & 2 & 0.0059 & 0.9896 & 0.8837 & 0.0233 & 0.7876 & 0.0351 & 0.7552 & 0.0261 \\
\hline & 4 & 0.0088 & 0.9844 & 0.9411 & 0.0280 & 0.8723 & 0.0280 & 0.8681 & 0.0295 \\
\hline & 6 & 0.0005 & 0.9999 & 0.9949 & 0.0034 & 0.9831 & 0.0073 & 0.8681 & 0.0295 \\
\hline & 8 & 0.0015 & 0.9983 & 0.9897 & 0.0066 & 0.9731 & 0.0146 & 0.9859 & 0.4663 \\
\hline \multirow[t]{4}{*}{ Hii et al } & 2 & 0.9739 & 0.0147 & 0.9999 & 0.0000 & 0.9999 & 0.0000 & 0.9957 & 0.0005 \\
\hline & 4 & 0.9963 & 0.0021 & 0.9651 & 0.0165 & 1.0000 & 0.0001 & 0.9999 & 0.0000 \\
\hline & 6 & 0.9947 & 0.0066 & 0.9999 & 0.0001 & 0.9999 & 0.0001 & 0.9434 & 0.0126 \\
\hline & 8 & 1.0000 & 0.0000 & 0.9999 & 0.0000 & 0.9998 & 0.0001 & 0.9998 & 0.0001 \\
\hline \multirow[t]{4}{*}{ Agbashalo } & 2 & 0.9757 & 0.0136 & 0.9642 & 0.0072 & 0.9377 & 0.0103 & 0.9102 & 0.0096 \\
\hline & 4 & 0.9871 & 0.0073 & 0.9522 & 0.0227 & 0.9619 & 0.0113 & 0.9449 & 0.0123 \\
\hline & 6 & 0.9964 & 0.0048 & 0.9834 & 0.0112 & 0.9757 & 0.0105 & 0.9449 & 0.0123 \\
\hline & 8 & 0.9962 & 0.0034 & 0.9979 & 0.0014 & 0.9867 & 0.0072 & 0.9848 & 0.0067 \\
\hline \multirow[t]{4}{*}{ Singh } & 2 & 0.7685 & 0.1307 & 0.1227 & 0.1757 & 0.2377 & 0.2051 & 0.4733 & 0.1572 \\
\hline & 4 & 0.7452 & 0.1445 & 0.4929 & 0.2408 & 0.4009 & 0.2167 & 0.1295 & 0.1944 \\
\hline & 6 & 0.9041 & 0.0445 & 0.8342 & 0.1118 & 0.6975 & 0.1309 & 0.1295 & 0.1944 \\
\hline & 8 & 0.9868 & 0.0119 & 0.8443 & 0.1003 & 0.6760 & 0.1751 & 0.7493 & 0.1108 \\
\hline \multirow[t]{4}{*}{ Demir et al } & 2 & 0.9966 & 0.0019 & 0.9995 & 0.0002 & 0.9997 & 0.0000 & 0.9998 & 0.0000 \\
\hline & 4 & 0.9952 & 0.0027 & 0.9961 & 0.0018 & 0.9990 & 0.0005 & 0.9974 & 0.0006 \\
\hline & 6 & 0.9983 & 0.0005 & 0.9976 & 0.0016 & 0.9989 & 0.0011 & 0.9974 & 0.0006 \\
\hline & 8 & 0.9990 & 0.0009 & 0.9999 & 0.0001 & 0.9999 & 0.0002 & 0.9988 & 0.0005 \\
\hline \multirow[t]{4}{*}{ Midilli et al } & 2 & 0.9976 & 0.0013 & 0.9957 & 0.0009 & 0.9974 & 0.0004 & 0.9944 & 0.9974 \\
\hline & 4 & 0.9443 & 0.0316 & 0.9941 & 0.0028 & 0.9968 & 0.0020 & 0.9950 & 0.9968 \\
\hline & 6 & 0.9806 & 0.0401 & 0.9995 & 0.0003 & 0.9948 & 0.0079 & 0.9935 & 0.9948 \\
\hline & 8 & 0.9994 & 0.0005 & 0.9999 & 0.0001 & 0.9979 & 0.0059 & 0.9846 & 0.9979 \\
\hline \multirow[t]{4}{*}{ Modified Midilli } & 2 & 0.9968 & 0.0018 & 0.9909 & 0.0018 & 0.9776 & 0.0037 & 0.9687 & 0.0033 \\
\hline & 4 & 0.9970 & 0.0017 & 0.9883 & 0.0056 & 0.9921 & 0.0031 & 0.9859 & 0.0031 \\
\hline & 6 & 0.9997 & 0.0002 & 0.9995 & 0.0003 & 0.9994 & 0.0006 & 0.9859 & 0.0031 \\
\hline & 8 & 0.9996 & 0.0004 & 0.9993 & 0.0005 & 0.9996 & 0.0008 & 0.9859 & 0.0003 \\
\hline \multirow[t]{4}{*}{ Henderson and Pabis } & 2 & 0.8669 & 0.0749 & 0.7357 & 0.0528 & 0.6494 & 0.0578 & 0.6212 & 0.0403 \\
\hline & 4 & 0.8917 & 0.0611 & 0.7455 & 0.1208 & 0.7217 & 0.0766 & 0.8817 & 0.0683 \\
\hline & 6 & 0.9665 & 0.0295 & 0.9024 & 0.0658 & 0.8499 & 0.0650 & 0.6940 & 0.0683 \\
\hline & 8 & 0.9885 & 0.0103 & 0.9362 & 0.0409 & 0.8879 & 0.0765 & 0.8810 & 0.0525 \\
\hline \multirow[t]{4}{*}{ Logarithmic } & 2 & 0.9966 & 0.0019 & 0.9991 & 0.0002 & 0.9997 & 0.0000 & 0.9999 & 0.0001 \\
\hline & 4 & 0.9952 & 0.0027 & 0.9988 & 0.0018 & 0.9981 & 0.0005 & 0.9999 & 0.0001 \\
\hline & 6 & 0.9992 & 0.0007 & 0.9976 & 0.0016 & 0.9974 & 0.0011 & 0.9997 & 0.0006 \\
\hline & 8 & 0.9990 & 0.0009 & 0.9995 & 0.0001 & 1.0000 & 0.0002 & 0.9999 & 0.0005 \\
\hline
\end{tabular}

From Table 2 it was observed that the statistical measurements of some selected models for drying sweet potatoes gave good fit, but when compared together Hii et al model gave higher values of coefficient of determination $\left(\mathrm{R}^{2}\right)$ of 0.9999 for the various thickness and different drying temperatures studied and was also found to have corresponding low values for sum of squares calculated as they tended towards zero. This observation was found to be in agreement with the findings of other researchers [12]. Singh model was the least among the fitted models.

The model with the highest value for $\mathrm{R}^{2}$ was selected to describe the drying curves, as well as the lowest of values of the Sum of squares error (SSE) gave the best fit.

The quality of the developed model has been evaluated using different statistical criteria. Table 3 shows that the value of $\mathrm{R}^{2}$ is 0.999963 while other error functions are RMSE 0.001022 , MBE -0.10673, SSE -0.000001055 and Chi square ( $\left.\chi^{2}\right) 0.000001044475$. The values of $\mathrm{R}^{2}$ and other statistical measures were better compared to the findings of several previous works in fitting the model to the experimental data $[4,9]$.

Table 3. The statistical values of the developed model for this study.

\begin{tabular}{llllll}
\hline $\mathbf{R}^{2}$ & RSS & RMSE & MBE & SSE & $\chi^{2}$ \\
\hline 0.999963 & 0.000999264 & 0.001022 & -0.10673 & $1.05519 \mathrm{E}-06$ & $1.04475 \mathrm{E}-06$ \\
\hline
\end{tabular}

However, two parameters $[12,40]$ were used to develop a model that relates moisture ratio (MR) to mass (M) and time (t) for drying sweet potato which this research work has been able to establish. These constants $(0.005263,0.991698$ and
0.002583 ) were obtained using solver (after the iteration with the GRG protocol).

The empirical model developed is given as Equation 16: 


$$
M R=0.005263\left[\frac{M^{0.991698}}{t^{0.002583}}\right]
$$

The individual constants of the selected thirteen models for the drying sweet potato in this study were presented in Table 4. When the rate constant is small, the evaporation rate is so small that the moisture content decreases very slowly [41]. Also, the drying coefficient gives a measure of the time required to dry out the moisture content from the material
[42]. From the results determined, the rate constants are very small (less than 1) which means the evaporation rates involved in drying sweet potato were slow apart from Hii et al model where $\mathrm{K}$ was up to 5 at $70^{\circ} \mathrm{C}$ for $2 \mathrm{~mm}$. Moreover, the other model constants for some thin layer drying kinetic models were small apart from Midilli and Hii et al where some constants were above 1 .

The application of these are useful engineering tool for the design of equipment and optimization.

Table 4. Selected Models with their Constants at different Temperatures and Thickness.

\begin{tabular}{|c|c|c|c|c|c|c|c|c|c|}
\hline \multirow{3}{*}{$\begin{array}{l}\text { Model } \\
\text { name }\end{array}$} & \multicolumn{9}{|c|}{ Temperature } \\
\hline & \multicolumn{2}{|c|}{$40^{\circ} \mathrm{C}$} & \multicolumn{3}{|l|}{$50^{\circ} \mathrm{C}$} & \multicolumn{2}{|l|}{$60^{\circ} \mathrm{C}$} & \multicolumn{2}{|l|}{$7^{\circ} \mathrm{C}$} \\
\hline & Thickness & Model constant & $\mathbf{R}^{2}$ & Model constant & $\mathbf{R}^{2}$ & Model constant & $\mathbf{R}^{2}$ & Model constant & $\mathbf{R}^{2}$ \\
\hline \multirow[t]{4}{*}{ Lewis } & 2 & $\mathrm{~K}=0.002136$ & 0.7680 & $\mathrm{~K}=0.004285$ & 0.1310 & $\mathrm{~K}=0.005194$ & 0.9050 & $\mathrm{~K}=0.007076$ & -0.4730 \\
\hline & 4 & $\mathrm{~K}=0.002015$ & 0.7450 & $\mathrm{~K}=0.003128$ & 0.4920 & $K=0.003462$ & 0.9100 & $K=0.005212$ & 0.1290 \\
\hline & 6 & $\mathrm{~K}=0.001536$ & 0.9490 & $\mathrm{~K}=0.002609$ & 0.3020 & $K=0.003449$ & 0.6990 & $\mathrm{~K}=0.005212$ & 0.1290 \\
\hline & 8 & $\mathrm{~K}=0.00123$ & 0.9870 & $\mathrm{~K}=0.001713$ & 0.8440 & $\mathrm{~K}=0.002607$ & 0.6790 & $\mathrm{~K}=0.003412$ & 0.5830 \\
\hline \multirow[t]{4}{*}{ Page } & 2 & $\begin{array}{l}\mathrm{K}=0.018752 \\
\mathrm{n}=0.632172\end{array}$ & 0.9350 & $\begin{array}{l}\mathrm{K}=0.077578 \\
\mathrm{n}=0.456215\end{array}$ & 0.8780 & $\begin{array}{l}\mathrm{K}=0.1108 \\
\mathrm{n}=0.411401\end{array}$ & 0.8220 & $\begin{array}{l}\mathrm{K}=0.138555 \\
\mathrm{n}=0.394533\end{array}$ & 0.7890 \\
\hline & 4 & $\begin{array}{l}K=0.021606 \\
n=0.603662\end{array}$ & 0.9570 & $\begin{array}{l}\mathrm{K}=0.04505 \\
\mathrm{n}=0.531598\end{array}$ & 0.9920 & $\begin{array}{l}\mathrm{K}=0.065875 \\
\mathrm{n}=0.467224\end{array}$ & 0.8700 & $\begin{array}{l}\mathrm{K}=0.087611 \\
\mathrm{n}=0.4611\end{array}$ & 0.8460 \\
\hline & 6 & $\begin{array}{l}\mathrm{K}=0.00562 \\
\mathrm{n}=0.790284\end{array}$ & 0.9970 & $\begin{array}{l}\mathrm{K}=0.017589 \\
\mathrm{n}=0.673063\end{array}$ & 0.9530 & $\begin{array}{l}\mathrm{K}=0.03179 \\
\mathrm{n}=0.601256\end{array}$ & 0.9290 & $\begin{array}{l}\mathrm{K}=0.087611 \\
\mathrm{n}=0.4611\end{array}$ & 0.8460 \\
\hline & 8 & $\begin{array}{l}\mathrm{K}=0.002294 \\
\mathrm{n}=0.90063\end{array}$ & 0.9920 & $\begin{array}{l}\mathrm{K}=0.013988 \\
\mathrm{n}=0.655464\end{array}$ & 0.9800 & $\begin{array}{l}\mathrm{K}=0.030142 \\
\mathrm{n}=0.580559\end{array}$ & 0.9430 & $\begin{array}{l}\mathrm{K}=0.028473 \\
\mathrm{n}=0.619668\end{array}$ & 0.9470 \\
\hline \multirow[t]{4}{*}{$\begin{array}{l}\text { Modified } \\
\text { page }\end{array}$} & 2 & $\begin{array}{l}\mathrm{K}=0.032679 \\
\mathrm{n}=0.065358\end{array}$ & 0.7680 & $\begin{array}{l}\mathrm{K}=0.031742 \\
\mathrm{n}=0.063485\end{array}$ & 0.7450 & $\begin{array}{l}\mathrm{K}=0.048044 \\
\mathrm{n}=0.108099\end{array}$ & -0.2370 & $\begin{array}{l}\mathrm{K}=0.059482 \\
\mathrm{n}=0.118964\end{array}$ & -0.4730 \\
\hline & 4 & $\begin{array}{l}\mathrm{K}=0.031742 \\
\mathrm{n}=0.063485\end{array}$ & 0.7450 & $\begin{array}{l}\mathrm{K}=0.033944 \\
\mathrm{n}=0.045259\end{array}$ & 0.9490 & $\begin{array}{l}\mathrm{K}=0.041604 \\
\mathrm{n}=0.083209\end{array}$ & 0.7980 & $\begin{array}{l}\mathrm{K}=0.051048 \\
\mathrm{n}=0.102097\end{array}$ & 0.1290 \\
\hline & 6 & $\begin{array}{l}\mathrm{K}=0.033944 \\
\mathrm{n}=0.045259\end{array}$ & 0.9490 & $\begin{array}{l}\mathrm{K}=0.045284 \\
\mathrm{n}=0.02717\end{array}$ & 0.9860 & $\begin{array}{l}\mathrm{K}=0.041525 \\
\mathrm{n}=0.083049\end{array}$ & 0.9030 & $\begin{array}{l}\mathrm{K}=0.051048 \\
\mathrm{n}=0.102097\end{array}$ & 0.1290 \\
\hline & 8 & $\begin{array}{l}\mathrm{K}=0.045284 \\
\mathrm{n}=0.02717 \\
\mathrm{a}=0.70346\end{array}$ & 0.9860 & $\begin{array}{l}\mathrm{K}=0.031742 \\
\mathrm{n}=0.063485 \\
\mathrm{a}=0.707484\end{array}$ & 0.7450 & $\begin{array}{l}K=0.036105 \\
n=0.072209 \\
a=0.813895\end{array}$ & 0.9600 & $\begin{array}{l}K=0.041302 \\
n=0.082604 \\
a=0.92369\end{array}$ & 0.7490 \\
\hline \multirow[t]{4}{*}{ Logarithmic } & 2 & $\begin{array}{l}K=0.00661 \\
c=0.366044 \\
a=0.652812\end{array}$ & 0.9960 & $\begin{array}{l}K=0.016705 \\
c=0.359283 \\
a=0.795417\end{array}$ & 0.9990 & $\begin{array}{l}K=0.023185 \\
c=0.341934 \\
a=0.718074\end{array}$ & 0.9990 & $\begin{array}{l}\mathrm{K}=0.032999 \\
\mathrm{c}=0.332617 \\
\mathrm{a}=0.116225\end{array}$ & 0.9990 \\
\hline & 4 & $\begin{array}{l}K=0.005429 \\
c=0.34956 \\
a=0.730995\end{array}$ & 0.9950 & $\begin{array}{l}K=0.010947 \\
c=0.323369 \\
a=0.760523\end{array}$ & 0.9980 & $\begin{array}{l}K=0.013953 \\
c=0.363798 \\
a=0.751903\end{array}$ & 0.9980 & $\begin{array}{l}K=0.008653 \\
c=0.308695 \\
a=0.814707\end{array}$ & 0.9990 \\
\hline & 6 & $\begin{array}{l}\mathrm{K}=0.003154 \\
\mathrm{c}=0.299462\end{array}$ & 0.9990 & $\begin{array}{l}\mathrm{K}=0.006265 \\
\mathrm{c}=0.289745\end{array}$ & 0.9980 & $\begin{array}{l}\mathrm{K}=0.009609 \\
\mathrm{c}=0.30976\end{array}$ & 0.9970 & $\begin{array}{l}\mathrm{K}=0.019199 \\
\mathrm{c}=0.311617\end{array}$ & 0.9990 \\
\hline & 8 & $\begin{array}{l}\mathrm{a}=0.767738 \\
\mathrm{~K}=0.002124 \\
\mathrm{c}=0.261943\end{array}$ & 0.9990 & $\begin{array}{l}\mathrm{a}=0.631224 \\
\mathrm{~K}=0.003879 \\
\mathrm{c}=0.338606\end{array}$ & 0.9990 & $\begin{array}{l}a=0.708956 \\
K=0.00728 \\
c=0.319596\end{array}$ & 0.9990 & $\begin{array}{l}a=0.73754 \\
K=0.008754 \\
c=0.301264\end{array}$ & 0.9990 \\
\hline \multirow[t]{4}{*}{$\begin{array}{l}\text { Henderson } \\
\text { and Pabis }\end{array}$} & 2 & $\begin{array}{l}a=0.851751 \\
K=0.00164\end{array}$ & 0.8660 & $\begin{array}{l}a=0.69215 \\
K=0.002304\end{array}$ & 0.7350 & $\begin{array}{l}a=0.63344 \\
K=0.002419\end{array}$ & 0.6490 & $\begin{array}{l}a=0.603394 \\
K=0.003036\end{array}$ & 0.6210 \\
\hline & 4 & $\begin{array}{l}a=0.827938 \\
K=0.001479\end{array}$ & 0.8910 & $\begin{array}{l}a=0.747359 \\
K=0.002005\end{array}$ & 0.7450 & $\begin{array}{l}a=0.708791 \\
K=0.001939\end{array}$ & 0.7210 & $\begin{array}{l}a=0.660201 \\
K=0.002738\end{array}$ & 0.8820 \\
\hline & 6 & $\begin{array}{l}a=0.934434 \\
K=0.001377\end{array}$ & 0.9660 & $\begin{array}{l}a=0.855444 \\
K=0.002082\end{array}$ & 0.9020 & $\begin{array}{l}\mathrm{a}=0.799534 \\
\mathrm{~K}=0.002473\end{array}$ & 0.8490 & $\begin{array}{l}a=0.660205 \\
K=0.002738\end{array}$ & 0.6940 \\
\hline & 8 & $\begin{array}{l}a=0.980114 \\
K=0.001187\end{array}$ & 0.9880 & $\begin{array}{l}a=0.864499 \\
K=0.001343\end{array}$ & 0.9360 & $\begin{array}{l}\mathrm{a}=0.793157 \\
\mathrm{~K}=0.001833\end{array}$ & 0.8870 & $\begin{array}{l}a=0.811336 \\
K=0.002507\end{array}$ & 0.8810 \\
\hline \multirow[t]{3}{*}{ Midilli } & 2 & $\begin{array}{l}\mathrm{a}=1.050136 \\
\mathrm{~K}=0.00528 \\
\mathrm{n}=0.951291 \\
\mathrm{~b}=0.000473 \\
\mathrm{a}=1.797361\end{array}$ & 0.9980 & $\begin{array}{l}\mathrm{a}=3.098222 \\
\mathrm{~K}=0.524682 \\
\mathrm{n}=0.284328 \\
\mathrm{~b}=0.00048 \\
\mathrm{a}=1.21959\end{array}$ & 0.9960 & $\begin{array}{l}\mathrm{a}=4.428267 \\
\mathrm{~K}=0.729246 \\
\mathrm{n}=0.266459 \\
\mathrm{~b}=0.00061 \\
\mathrm{a}=2.524963\end{array}$ & 0.9970 & $\begin{array}{l}a=5.989524 \\
K=0.896964 \\
n=0.266202 \\
b=0.000878 \\
a=1.727501\end{array}$ & 0.9940 \\
\hline & 4 & $\begin{array}{l}\mathrm{K}=0.312868 \\
\mathrm{n}=0.256263 \\
\mathrm{~b}=3.96 \mathrm{E}-05 \\
\mathrm{a}=1.78469\end{array}$ & 0.9440 & $\begin{array}{l}\mathrm{K}=0.02685 \\
\mathrm{n}=0.737562 \\
\mathrm{~b}=0.000478 \\
\mathrm{a}=1.013301\end{array}$ & 0.9940 & $\begin{array}{l}\mathrm{K}=0.372856 \\
\mathrm{n}=0.320272 \\
\mathrm{~b}=0.000415 \\
\mathrm{a}=3.560496\end{array}$ & 0.9970 & $\begin{array}{l}\mathrm{K}=0.15133 \\
\mathrm{n}=0.502302 \\
\mathrm{~b}=0.000626 \\
\mathrm{a}=3.690931\end{array}$ & 0.9950 \\
\hline & 6 & $\begin{array}{l}\mathrm{K}=0.217979 \\
\mathrm{n}=0.294226\end{array}$ & 0.9810 & $\begin{array}{l}\mathrm{K}=0.003946 \\
\mathrm{n}=1.014267\end{array}$ & 0.9990 & $\begin{array}{l}\mathrm{K}=0.606871 \\
\mathrm{n}=0.242032\end{array}$ & 0.9950 & $\begin{array}{l}\mathrm{K}=0.571427 \\
\mathrm{n}=0.299485\end{array}$ & 0.9930 \\
\hline
\end{tabular}




\begin{tabular}{|c|c|c|c|c|c|c|c|c|c|}
\hline \multirow{3}{*}{$\begin{array}{l}\text { Model } \\
\text { name }\end{array}$} & \multicolumn{9}{|c|}{ Temperature } \\
\hline & \multicolumn{2}{|c|}{$40^{\circ} \mathrm{C}$} & \multicolumn{3}{|l|}{$50^{\circ} \mathrm{C}$} & \multicolumn{2}{|l|}{$60^{\circ} \mathrm{C}$} & \multicolumn{2}{|l|}{$70^{\circ} \mathrm{C}$} \\
\hline & Thickness & Model constant & $\mathbf{R}^{2}$ & Model constant & $\mathbf{R}^{2}$ & Model constant & $\mathbf{R}^{2}$ & Model constant & $\mathbf{R}^{2}$ \\
\hline \multirow{17}{*}{$\begin{array}{l}\text { Modified } \\
\text { Midilli }\end{array}$} & \multirow{4}{*}{ - } & $\mathrm{b}=2.11 \mathrm{E}-05$ & & $\mathrm{~b}=0.0004$ & & $\mathrm{~b}=0.000149$ & & $\mathrm{~b}=0.000512$ & \\
\hline & & $a=1.024667$ & \multirow{3}{*}{0.9990} & $\mathrm{a}=1.033973$ & \multirow{3}{*}{0.9990} & $\mathrm{a}=2.288927$ & \multirow{3}{*}{0.9980} & $\mathrm{a}=3.683419$ & \multirow{3}{*}{0.9850} \\
\hline & & $K=0.001628$ & & $K=0.006937$ & & $K=0.332296$ & & $K=0.664916$ & \\
\hline & & $\begin{array}{l}\mathrm{n}=1.007941 \\
\mathrm{~b}=0.000182\end{array}$ & & $\begin{array}{l}\mathrm{n}=0.836983 \\
\mathrm{~b}=0.000265\end{array}$ & & $\begin{array}{l}\mathrm{n}=0.296599 \\
\mathrm{~b}=0.000119\end{array}$ & & $\begin{array}{l}\mathrm{n}=0.222597 \\
\mathrm{~b}=7.6 \mathrm{E}-05\end{array}$ & \\
\hline & \multirow{4}{*}{2} & $\mathrm{~K}=0.00294$ & \multirow{3}{*}{0.9970} & $\mathrm{~K}=0.016046$ & & $\mathrm{~K}=0.021219$ & & $\mathrm{~K}=0.025195$ & \\
\hline & & $\mathrm{n}=1.051711$ & & $\mathrm{n}=0.862239$ & 0.9910 & $\mathrm{n}=0.844273$ & 0.9780 & $\mathrm{n}=0.865174$ & 0.9690 \\
\hline & & $b=0.000504$ & & $b=0.000812$ & & $\mathrm{~b}=0.000887$ & & $\mathrm{~b}=0.001176$ & \\
\hline & & $\mathrm{K}=0.004948$ & & $K=0.006285$ & & $K=0.011862$ & & $\mathrm{~K}=0.015528$ & \\
\hline & 4 & $\mathrm{n}=0.934499$ & 0.9970 & $\mathrm{n}=0.98656$ & 0.9880 & $\mathrm{n}=0.889376$ & 0.9920 & $\mathrm{n}=0.90192$ & 0.9860 \\
\hline & & $\mathrm{b}=0.000387$ & & $\mathrm{~b}=0.000544$ & & $\mathrm{~b}=0.000695$ & & $\mathrm{~b}=0.000783$ & \\
\hline & & $K=0.001748$ & & $K=0.00337$ & & $K=0.005872$ & & $\mathrm{~K}=0.015528$ & \\
\hline & 6 & $\mathrm{n}=1.047153$ & 0.9990 & $\mathrm{n}=1.041626$ & 0.9990 & $\mathrm{n}=1.00206$ & 0.9990 & $\mathrm{n}=0.901919$ & 0.9860 \\
\hline & & $\mathrm{b}=0.000273$ & & $\mathrm{~b}=0.000407$ & & $\mathrm{~b}=0.000569$ & & $\mathrm{~b}=0.000783$ & \\
\hline & & $\mathrm{K}=0.001044$ & & $\mathrm{~K}=0.005111$ & & $\mathrm{~K}=0.006548$ & & $\mathrm{~K}=0.006388$ & \\
\hline & 8 & $\mathrm{n}=1.083479$ & 0.9990 & $\mathrm{n}=0.885295$ & 0.9990 & $\mathrm{n}=0.927841$ & 0.9990 & $\mathrm{n}=0.975357$ & 0.9990 \\
\hline & & $\mathrm{b}=0.000214$ & & $\mathrm{~b}=0.000282$ & & $\mathrm{~b}=0.000416$ & & $\mathrm{~b}=0.000521$ & \\
\hline & & $a=0.703462$ & & $a=0.707487$ & & $a=0.813896$ & & $\mathrm{a}=0.982221$ & \\
\hline & & $\mathrm{k}=0.087816$ & & $\mathrm{k}=0.120899$ & & $\mathrm{k}=0.162781$ & & $\mathrm{k}=0.172117$ & \\
\hline Demir et al & 2 & $\mathrm{n}=0.075271$ & $0.99 / 0$ & $\mathrm{n}=0.13817$ & 0.9990 & $\mathrm{n}=0.142433$ & 0.9990 & $\mathrm{n}=0.200803$ & 0.9990 \\
\hline & & $b=0.366046$ & & $b=0.359282$ & & $b=0.341934$ & & $b=0.334773$ & \\
\hline & & $a=0.652814$ & & $a=0.795415$ & & $a=0.718074$ & & $\mathrm{a}=0.814706$ & \\
\hline & & $\mathrm{k}=0.079586$ & & $\mathrm{k}=0.090611$ & & $\mathrm{k}=0.139763$ & & $\mathrm{k}=0.195952$ & \\
\hline & 4 & $\mathrm{n}=0.068217$ & 0.9950 & $\mathrm{n}=0.12081$ & 0.9960 & $\mathrm{n}=0.099831$ & 0.9990 & $\mathrm{n}=0.097976$ & 0.9970 \\
\hline & & $b=0.349562$ & & $b=0.323369$ & & $\mathrm{~b}=0.363798$ & & $\mathrm{~b}=0.311617$ & \\
\hline & & $a=0.737187$ & & $a=0.760524$ & & $a=0.751901$ & & $\mathrm{a}=0.814707$ & \\
\hline & 6 & $\mathrm{k}=0.180402$ & 0.9980 & $\mathrm{k}=0.084618$ & 0.9980 & $\mathrm{k}=0.089486$ & 0.9990 & $\mathrm{k}=0.107327$ & 0.9970 \\
\hline & 0 & $\mathrm{n}=0.01804$ & 0.9900 & $\mathrm{n}=0.074041$ & 0.9900 & $\mathrm{n}=0.107383$ & 0.9990 & $\mathrm{n}=0.178879$ & 0.9910 \\
\hline & & $b=0.304659$ & & $\mathrm{~b}=0.289745$ & & $\mathrm{~b}=0.30976$ & & $\mathrm{~b}=0.311617$ & \\
\hline & & $a=0.767729$ & & $a=0.651503$ & & $a=0.708958$ & & $\mathrm{a}=0.73754$ & \\
\hline & 8 & $\mathrm{k}=0.043108$ & 0.9990 & $\mathrm{k}=0.060404$ & & $\mathrm{k}=0.246302$ & & $\mathrm{k}=0.100022$ & 09990 \\
\hline & & $\mathrm{n}=0.049266$ & 0.9990 & $\mathrm{n}=0.069033$ & 0.9990 & $\mathrm{n}=0.029556$ & 0.9990 & $\mathrm{n}=0.087519$ & 0.9990 \\
\hline & & $b=0.261953$ & & $\mathrm{~b}=0.345808$ & & $b=0.319596$ & & $b=0.301264$ & \\
\hline & & $a=0.703463$ & & $a=0.707484$ & & $\mathrm{a}=0.339387$ & & $\mathrm{a}=0.982216$ & \\
\hline & & $\mathrm{K}_{0}=0.00661$ & & $\mathrm{~K}_{0}=0.016705$ & & $\mathrm{~K}_{0}=4.86 \mathrm{E}-07$ & & $\mathrm{~K}_{0}=0.034562$ & \\
\hline Two Term & 2 & $\mathrm{~b}=0.366046$ & 0.9980 & $\mathrm{~b}=0.359282$ & 0.9960 & $\mathrm{~b}=0.711025$ & 0.9950 & $\mathrm{~b}=0.334773$ & 0.9990 \\
\hline & & $\mathrm{K}_{1}=0$ & & $\mathrm{~K}_{1}=0$ & & $\mathrm{~K}_{1}=0.020375$ & & $\mathrm{~K}_{1}=0$ & \\
\hline & & $\mathrm{a}=0.353184$ & & $\mathrm{a}=0.795408$ & & $\mathrm{a}=0.718074$ & & $\mathrm{a}=0.814711$ & \\
\hline & & $\mathrm{K}_{0}=7.62 \mathrm{E}-07$ & & $\mathrm{~K}_{0}=0.010947$ & & $\mathrm{~K}_{0}=0.013953$ & & $\mathrm{~K}_{0}=0.019199$ & \\
\hline & 4 & $\mathrm{~b}=0.659939$ & 0.9950 & $\mathrm{~b}=0.323368$ & 0.9960 & $\mathrm{~b}=0.363798$ & 0.9990 & $\mathrm{~b}=0.311617$ & 0.9970 \\
\hline & & $\mathrm{K}_{1}=0.005598$ & & $\mathrm{~K}_{1}=0$ & & $\mathrm{~K}_{1}=0$ & & $\mathrm{~K}_{1}=0$ & \\
\hline & & $\mathrm{a}=0.299455$ & & $a=0.289739$ & & $a=0.799531$ & & $\mathrm{a}=0.311618$ & \\
\hline & 6 & $\begin{array}{l}\mathrm{K}_{0}=0 \\
\mathrm{~h}=0731\end{array}$ & 0.9980 & $\mathrm{~K}_{0}=0$ & 0.9980 & $\mathrm{~K}_{0}=0.002473$ & 0.9350 & $\mathrm{~K}_{0}=0$ & 0.9970 \\
\hline & & $\begin{array}{l}\mathrm{b}=0.731 \\
\mathrm{~K}_{1}=0.003154\end{array}$ & & $\begin{array}{l}b=0.760512 \\
K_{1}=0.006265\end{array}$ & & $\begin{array}{l}\mathrm{b}=0.008 \\
\mathrm{~K}_{1}=1.155609\end{array}$ & & $\begin{array}{l}\mathrm{b}=0.81471 \\
\mathrm{~K}_{1}=0.019199\end{array}$ & \\
\hline & & $\mathrm{a}=0.008$ & & $\mathrm{a}=0.364922$ & & $\mathrm{a}=0.319592$ & & $\mathrm{a}=0.737536$ & \\
\hline & 8 & $\mathrm{~K}_{0}=0.98011$ & 0.9890 & $\mathrm{~K}_{0}=6.11 \mathrm{E}-05$ & 09990 & $\mathrm{~K}_{0}=0$ & 09990 & $\mathrm{~K}_{0}=0.008754$ & 09990 \\
\hline & 8 & $\mathrm{~b}=0.001187$ & 0.9890 & $\mathrm{~b}=0.634302$ & 0.9990 & $b=0.708948$ & 0.9990 & $\mathrm{~b}=0.301264$ & 0.9990 \\
\hline & & $\mathrm{K}_{1}=0.010553$ & & $\mathrm{~K}_{1}=0.004312$ & & $\mathrm{~K}_{1}=0.007279$ & & $\mathrm{~K}_{1}=0$ & \\
\hline Singh & 2 & $\begin{array}{l}\mathrm{K}=0.002136 \\
\mathrm{a}=0\end{array}$ & 0.7680 & $\begin{array}{l}K=0.004374 \\
a=0\end{array}$ & 0.1227 & $\begin{array}{l}K=0.005194 \\
a=0\end{array}$ & -0.2380 & $\begin{array}{l}\mathrm{K}=0.007076 \\
\mathrm{a}=4.83 \mathrm{E}-07\end{array}$ & -0.4730 \\
\hline & 4 & $\begin{array}{l}K=0.002015 \\
a=0\end{array}$ & 0.7450 & $\begin{array}{l}K=0.003128 \\
a=0\end{array}$ & 0.4930 & $\begin{array}{l}K=0.003462 \\
a=0\end{array}$ & -0.4010 & $\begin{array}{l}K=0.004 \\
a=0.005212\end{array}$ & 0.1300 \\
\hline & 6 & $\begin{array}{l}K=0.001536 \\
a=0\end{array}$ & 0.9040 & $\begin{array}{l}K=0.002609 \\
a=0\end{array}$ & 0.8340 & $\begin{array}{l}K=0.003537 \\
a=0\end{array}$ & 0.6970 & $\begin{array}{l}\mathrm{K}=0.005212 \\
\mathrm{a}=0\end{array}$ & 0.1300 \\
\hline & 8 & $\begin{array}{l}\mathrm{K}=0.00123 \\
\mathrm{a}=0\end{array}$ & 0.9870 & $\begin{array}{l}K=0.001713 \\
a=0\end{array}$ & 0.8440 & $\begin{array}{l}K=0.002685 \\
a=0\end{array}$ & 0.6760 & $\begin{array}{l}\mathrm{K}=0.003412 \\
\mathrm{a}=0\end{array}$ & 0.7490 \\
\hline Agbashalo & 2 & $\mathrm{~K}_{1}=0.004263$ & 0.9760 & $\mathrm{~K}_{1}=0.012863$ & 0.9640 & $\mathrm{~K}_{1}=0.017325$ & 0.9380 & $\mathrm{~K}_{1}=0.023411$ & 0.9100 \\
\hline Agoasnalo & 2 & $\mathrm{~K}_{2}=0.002529$ & 0.9100 & $\mathrm{~K}_{2}=0.009147$ & 0.9040 & $\mathrm{~K}_{2}=0.012091$ & 0.9580 & $\mathrm{~K}_{2}=0.015938$ & 0.9100 \\
\hline & 4 & $\mathrm{~K}_{1}=0.004213$ & 0.9870 & $\mathrm{~K}_{1}=0.007871$ & 0.9520 & $\mathrm{~K}_{1}=0.010318$ & 0.9620 & $\mathrm{~K}_{1}=0.014987$ & 0.9450 \\
\hline & 4 & $\mathrm{~K}_{2}=0.002563$ & $0.98 / 0$ & $\mathrm{~K}_{2}=0.004711$ & 0.9520 & $\mathrm{~K}_{2}=0.007375$ & 0.9020 & $\mathrm{~K}_{2}=0.009242$ & 0.9450 \\
\hline & 6 & $\begin{array}{l}K_{1}=0.002194 \\
K_{2}=0000818\end{array}$ & 0.9960 & $\begin{array}{l}\mathrm{K}_{1}=0.004682 \\
K_{2}=0.002128\end{array}$ & 0.9830 & $\mathrm{~K}_{1}=0.007211$ & 0.9760 & $\mathrm{~K}_{1}=0.014988$ & 0.9450 \\
\hline & & & & & & & & & \\
\hline & 8 & $\begin{array}{l}\mathrm{K}_{1}=0.00146 \\
\mathrm{~K}_{2}=0.000327\end{array}$ & 0.9960 & $\begin{array}{l}\mathrm{K}_{1}=0.003177 \\
\mathrm{~K}_{2}=0.0018\end{array}$ & 0.9980 & $\begin{array}{l}K_{1}=0.005782 \\
K_{2}=0.003296\end{array}$ & 0.9870 & $\begin{array}{l}K_{1}=0.006815 \\
K_{2}=0.003474\end{array}$ & 0.9850 \\
\hline
\end{tabular}




\begin{tabular}{|c|c|c|c|c|c|c|c|c|c|}
\hline \multirow{3}{*}{$\begin{array}{l}\text { Model } \\
\text { name }\end{array}$} & \multicolumn{9}{|c|}{ Temperature } \\
\hline & \multicolumn{2}{|c|}{$40^{\circ} \mathrm{C}$} & \multicolumn{3}{|l|}{$50^{\circ} \mathrm{C}$} & \multicolumn{2}{|l|}{$60^{\circ} \mathrm{C}$} & \multicolumn{2}{|l|}{$7^{\circ} \mathrm{C}$} \\
\hline & Thickness & Model constant & $\mathbf{R}^{2}$ & Model constant & $\mathbf{R}^{2}$ & Model constant & $\mathbf{R}^{2}$ & Model constant & $\mathbf{R}^{2}$ \\
\hline \multirow[t]{4}{*}{ Hii et al } & 2 & $\begin{array}{l}\mathrm{a}=0.041907 \\
\mathrm{~K}_{1}=0.257035 \\
\mathrm{n}=0.410749 \\
\mathrm{~b}=2.343353 \\
\mathrm{~K}_{2}=0.398378 \\
\mathrm{n}=0.241939\end{array}$ & 0.9740 & $\begin{array}{l}a=0.733742 \\
K_{1}=0.702389 \\
n=9.35 E-08 \\
b=0.600552 \\
K_{2}=0.005764 \\
n=1.212526\end{array}$ & 0.9990 & $\begin{array}{l}\mathrm{a}=0.755104 \\
\mathrm{~K}_{1}=0.016358 \\
\mathrm{n}=1.071261 \\
\mathrm{~b}=0.475716 \\
\mathrm{~K}_{2}=0.323469 \\
\mathrm{n}=0.001803\end{array}$ & 0.9990 & $\begin{array}{l}a=52.86507 \\
K_{1}=5.094837 \\
n=0 \\
b=76.38476 \\
K_{2}=2.187207 \\
n=0.263271\end{array}$ & 0.9960 \\
\hline & 4 & $\begin{array}{l}\mathrm{a}=0.745248 \\
\mathrm{~K}_{1}=0.716233 \\
\mathrm{n}=0 \\
\mathrm{~b}=0.583894 \\
\mathrm{~K}_{2}=0.00174 \\
\mathrm{n}=1.204611\end{array}$ & 0.9960 & $\begin{array}{l}a=70.02726 \\
K_{1}=5.928962 \\
n=1.69 E-06 \\
b=70.06436 \\
K_{2}=3.096805 \\
n=0.113728\end{array}$ & 0.9650 & $\begin{array}{l}\mathrm{a}=0.534108 \\
\mathrm{~K}_{1}=0.002076 \\
\mathrm{n}=1.384415 \\
\mathrm{~b}=0.692542 \\
\mathrm{~K}_{2}=0.441938 \\
\mathrm{n}=0.05881\end{array}$ & 0.9990 & $\begin{array}{l}\mathrm{a}=0.631275 \\
\mathrm{~K}_{1}=0.689391 \\
\mathrm{n}=0 \\
\mathrm{~b}=0.617217 \\
\mathrm{~K}_{2}=0.002966 \\
\mathrm{n}=1.378516\end{array}$ & 0.9990 \\
\hline & 6 & $\begin{array}{l}\mathrm{a}=1.167105 \\
\mathrm{~K}_{1}=0.022845 \\
\mathrm{n}=0.595909 \\
\mathrm{~b}=0.494017 \\
\mathrm{~K}_{2}=0.912311 \\
\mathrm{n}=0.608096\end{array}$ & 0.9990 & $\begin{array}{l}\mathrm{a}=0.454973 \\
\mathrm{~K}_{1}=0.386237 \\
\mathrm{n}=2.14 \mathrm{E}-09 \\
\mathrm{~b}=0.65654 \\
\mathrm{~K}_{2}=0.001315 \\
\mathrm{n}=1.286032\end{array}$ & 0.9990 & $\begin{array}{l}\mathrm{a}=0.623595 \\
\mathrm{~K}_{1}=0.001812 \\
\mathrm{n}=1.318813 \\
\mathrm{~b}=0.375357 \\
\mathrm{~K}_{2}=0.119222 \\
\mathrm{n}=0.038132\end{array}$ & 0.9990 & $\begin{array}{l}\mathrm{a}=0.3 \\
\mathrm{~K}_{1}=4 \\
\mathrm{n}=0.9 \\
\mathrm{~b}=18670387 \\
\mathrm{~K}_{2}=15.78937 \\
\mathrm{n}=0.02252\end{array}$ & 0.9430 \\
\hline & 8 & $\begin{array}{l}\mathrm{a}=0.661317 \\
\mathrm{~K}_{1}=0.000662 \\
\mathrm{n}=1.213715 \\
\mathrm{~b}=0.332816 \\
\mathrm{~K}_{2}=1.35 \mathrm{E}-08 \\
\mathrm{n}=0.274594\end{array}$ & 0.9990 & $\begin{array}{l}\mathrm{a}=0.341803 \\
\mathrm{~K}_{1}=8.71 \mathrm{E}-09 \\
\mathrm{n}=0.034157 \\
\mathrm{~b}=0.662946 \\
\mathrm{~K}_{2}=0.004822 \\
\mathrm{n}=0.973731\end{array}$ & 0.9990 & $\begin{array}{l}a=0.479353 \\
K_{1}=0.39277 \\
n=0 \\
b=0.678277 \\
K_{2}=0.004991 \\
n=1.069026\end{array}$ & 0.9990 & $\begin{array}{l}\mathrm{a}=0.38943 \\
\mathrm{~K}_{1}=0.220181 \\
\mathrm{n}=0 \\
\mathrm{~b}=0.656199 \\
\mathrm{~K}_{2}=0.003152 \\
\mathrm{n}=1.19391\end{array}$ & 0.9990 \\
\hline \multirow[t]{3}{*}{$\begin{array}{l}\text { Two Term } \\
\text { Exponential }\end{array}$} & 2 & $\begin{array}{l}a=0.961616 \\
K=0.003258\end{array}$ & 0.9900 & $\begin{array}{l}a=0.972854 \\
K=0.006777\end{array}$ & 0.8840 & $\begin{array}{l}a=0.978959 \\
K=0.008076\end{array}$ & 0.7880 & $\begin{array}{l}a=0.980516 \\
K=0.010889\end{array}$ & 0.7550 \\
\hline & 4 & $\begin{array}{l}a=0.963668 \\
K=0.00304\end{array}$ & 0.9840 & $\begin{array}{l}a=0.978283 \\
K=0.004671\end{array}$ & 0.9410 & $\begin{array}{l}a=0.972135 \\
K=0.005516\end{array}$ & 0.8720 & $\begin{array}{l}a=0.982418 \\
K=0.007707\end{array}$ & 0.8680 \\
\hline & 6 & $\begin{array}{l}a=0.963138 \\
K=0.002044\end{array}$ & 0.9990 & $\begin{array}{l}\mathrm{a}=0.975267 \\
\mathrm{~K}=0.003612\end{array}$ & 0.9950 & $\begin{array}{l}a=0.97568 \\
K=0.004977\end{array}$ & 0.9830 & $\begin{array}{l}a=0.982418 \\
K=0.007707\end{array}$ & 0.8680 \\
\hline
\end{tabular}

The experimental moisture ratio was plotted against the predicted moisture ratio for all the temperatures and thickness studied as represented in Figure 3, this was to validate the developed model with experimental moisture ratio data. The $\mathrm{R}^{2}$ was found to be unity which indicated the closeness of the data obtained from the model developed for this study to the experimental moisture ratio data.

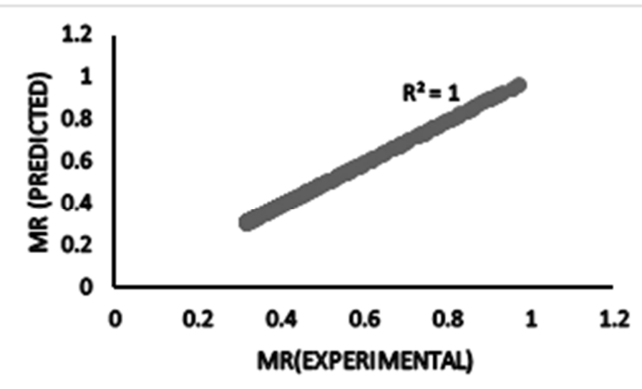

Figure 3. Experimental Moisture Ratio versus Predicted Moisture Ratio.

\section{Conclusion}

Drying process of sweet potato slices was investigated using a hot air oven dryer. A drying model showing the relationship between moisture ratio, drying time and mass on sweet potato drying process was developed. The statistical measures used for this study indicate that the derived model was in good agreement with experimental moisture ratio data. The developed model was fitted to experimental data and the coefficient of determination value obtained was 0.9999 and the sum of squares error value was 0.0001296 . The developed model was further validated by comparing the predicted moisture ratio against the experimental moisture data. The data points were identified to lie on a straight line, showing the suitability of the model in describing the drying kinetics of sweet potato at a temperature range of $40^{\circ} \mathrm{C}$ to $70^{\circ} \mathrm{C}$ and a sample thickness of $2 \mathrm{~mm}$ to $8 \mathrm{~mm}$.

Thirteen different kinetic models were compared with the developed model in order to determine the best model for predicting the drying kinetics of sweet potato, the drying rate was experimentally investigated at different temperature and material thickness levels. The experimental data were fitted to the thirteen selected thin layer models and the results obtained showed that the developed model resulted in an excellent fit for all drying temperatures of 40, 50, 60 and $70^{\circ} \mathrm{C}$ and sample thickness levels of 2, 4, 6 and $8 \mathrm{~mm}$. This result clearly shows that the developed model was suitable for predicting the drying of sweet potato. At all drying temperatures tested, the value of $R^{2}$ was higher than 0.9999 and the SSE value tended towards zero. Therefore, the developed model can be applied in describing the drying behaviour and predicting the drying kinetics of sweet potato and this developed model could be compared to Hii et al and Logarithmic models which had good $\mathrm{R}^{2}$ with least SSE; and 
can be useful in engineering tool for the application in the design of equipment and optimization.

\section{References}

[1] Kabiru, A. A., Joshua, A. A. and Raji, A. O. (2013) Effect of Slice Thickness and Temperature on the Drying Kinetics of Mango (Mangifera Indica), IJRRAS, 15 (1):41 - 50.

[2] Inyang, U. E., Oboh, I. O. and Etuk, B. R. (2018) Kinetic Models for Drying Techniques - Food Materials, Advances in Chemical Engineering and Science, 8(2): 27 - 48.

[3] Zhu, A. and Jiang, F. (2014). Modeling Of Mass Transfer Performance of Hot-Air Drying of Sweet Potato (Ipomoea Batatas L.) Slices, Chemical Industry and Chemical Engineering Quarterly, 20(2):171-181.

[4] Ndukwu M. C. and Nwabuisi, K. (2011) Thin Layer drying kinetics of varieties of cocoyam corm slice with heated air, Proceedings of the 11th International Conference and 32nd Annual General Meeting of the Nigerian Institution of Agricultural Engineers (NIAE Ilorin 2011), October 17 - 20, 2011, Ilorin, Nigeria, Vol. 32: 347 - 353.

[5] Carvalho, W. T., Oliveira, T. F., Silva, F. A., Caliari, M. and Soares jnr, M. S.,(2014) Drying kinetics of potato pulp waste, Food Science and Technology, Campinas, 34(1): 116-122.

[6] Aghbashlo, M. Kianmehr, M. H. and Arabhosseini, A.(2009) Modeling of thin-layer drying of potato slices in length of continuous band dryer, Energy Conversion and Management, 50:1348-1355.

[7] Hossain, M. A. and Bala, B. K. (2007). Drying of hot Chili Using Solar Tunnel Drier, Solar Energy, 18: 85-92.

[8] Fortes, M. and Okos, M. R. (1981). A non-equilibrium thermodynamics approach to transport phenomena in capillary porous media, Trans of ASAE, St Joseph, 24(3):756 -760.

[9] Ayim, I., Amankwah, E. A. and Dzisi, K. A. (2012) Effect of pretreatment and temperature on the air drying of French and False horn plantain slices. Journal of Animal and Plant Sciences, 13(2):1771-1780.

[10] Omolola, A. O., Jideani, A. I. O. and Kapila, P. F. (2015) Drying Kinetics of Banana (Musa Spp.). Interciencia, 40, 374380 .

[11] Sridhar, D. and Madhu, G. M.(2015) Drying kinetics and mathematical modeling of casuarinas Equisetifolia wood chips at various temperatures, Periodica Polytechnica chemical Engineering, 59(4):288 - 295.

[12] Onwude, D. I., Hanshim, N., Janius, R. B., Nawi, N. M. and Abdan, K. (2016) Modeling the Thin Layer Drying of Fruits and Vegetables: A Review. Comprehensive Reviews in Food Science and Food Safety, 15, 299-618.

[13] ASAE (2005), 'Manure Production and Characteristics', ASAE Standard, D384.2, American Society of Agricultural Engineers, St. Joseph, Michigan, USA.

[14] Akpinar, E. K. (2006). Determination of suitable thin layer drying curve model for some vegetables and fruits. Journal of Food Engineering 73: 75-84.

[15] Ronoh, E. K., Kanali, C. L., Mailutha, J. T. and Shitanda, D.
2009. Modeling thin layer drying of amaranth seeds under open sun and natural convection solar tent dryer. Agricultural Engineering International: The CIGR Ejournal XI: Manuscript 1420.

[16] Doymaz,. I. (2004). Convective air drying characteristics of thin layer carrots. Journal of Food Engineering. 61:359-364.

[17] Kingsly, A. R. P. and Singh, D. B. (2007). Drying kinetics of pomegranate arils. Journal of Food Engineering 79: 741- 744.

[18] Saeed, I. E., Sopian, K. and Zainol Abidin, Z. (2006) Drying Kinetics of Roselle (Hibiscus sabdariffa L.): Dried in Constant Temperature and Humidity Chamber. In: Muchtar, Ed., Proceedings of SPS, Permata, Bangi, S. D. E., Malaysia, 143148.

[19] Amer, B. A., M. A. Morcos, M. A. and Sabbah, M. A. (2003) New Method for the Mathematical Determination of Drying rates of Fig Fruits Depending on Empirical Data under conditions Suiting Solar Drying, conference Deutshcher Tropentag "Technological and institutional innovations for sustainable rural development" at Gottingen Germany, October 2003, Pp 1-13.

[20] Amer, B. A., M. A. Morcos, M. A. and Sabbah, M. A. (1999) New Method for the Mathematical Determination of Drying rates of Fig Fruits Depending on Empirical Data under conditions Suiting Solar Drying, Pp 1-13 In: Amer, B. M. A. (1999). Determination of Drying Rate of Fruits as a Function of the affecting factors under Conditions Suiting Solar Drying. M. Sc. Thesis, Ag. Eng. Dept., Fac. of Agic., Cairo Univ., Egypt.

[21] Khazaei, J. and Daneshmandi, S. (2007) Modeling of ThinLayer Drying Kinetics of Sesame Seeds: Mathematical and Neural Networking Modeling. International Agrophysics, 21, 335-348.

[22] Maisnam, D., Rasane, P., Dey, A., kaur, S. and Sarma, C. (2017) Recent Advances in Conventional Drying of Foods. Journal of Food Technology and Preservation, 1:25-34.

[23] Wei, J. (2013). Multivariate Numerical Optimization. Lecture Note. https://studentportalen.uu.se/uusp-filearea-tool. Accessed: 1st June, 2018.

[24] Smyth, G. K. (2015). Optimization and Nonlinear Equations. Wiley StataRef: Statistics Reference Online. pp. 1-9. doi: 10.1002/9781118445112.stat05030.pub2

[25] Pashaie, M., Sadeghi, M. and Jafarian, A. (2016). Artificial Neural Networks with Nelder-Mead Optimization Method for solving Nonlinear Integral Equations. Journal of Computer Science and Application, 8(1): 1-20.

[26] Okon, A. N., Udoh, F. D. and Appah, D. (2015). Empirical Wellhead Pressure - Production Rate Correlations for Niger Delta Oil Wells. Paper presented at the Society of Petroleum Engineers Nigeria Annual International Conference and Exhibition, Lagos, Nigeria, 4-6 August, pp. 1-17.

[27] Westerman, P. W., White, G. M. and Ross, I. J. (1973) Relative Humidity Effect on the High Temperature Drying of Shelled Corn. Transactions of the ASAE, 16, 1136-1139.

[28] Page, G. E. (1949) Factors Influencing the Maximum Rate of Air Drying Shelled Corn in Thin-Layers. M. S. Thesis, Purdue University, West Lafayette. 
[29] Yaldiz, O., Ertekin, C. and Uzun, H. B. (2001) Mathematical Modelling of Thin Layer Solar Drying of Sultana Grapes. Energy, 26, 457-465.

[30] Yagcioglu, A., Degirmencioglu, A. and Cagatay, F. (1999) Drying Characteristics of Laurel Leaves under Different Conditions. Proceedings of the 7th International Congress on Agricultural Mechanization and Energy, Adana, 26-27 May 1999, 565-569.

[31] Yaldiz, O. and Ertekin, C. (2001) Thin Layer Solar Drying of Some Vegetables. Drying Technology, 19, 583-597.

[32] Rahman, M. S., Perera, C. and Thebaud, C. (1998) Desorption Isotherm and Heat Pump Drying Kinetics of Peas. Journal of food research International, 30, 485-491.

[33] Singh, F., Katiyar, V. K. and Singh, B. P. (2014) Mathematical Modeling to Study Drying Characteristic of Apple and Potato. International Conference on Chemical, Environment and Biological Sciences, Kuala Lumpur, 17-18 September 2014, 172-175.

[34] Aghabashlo, M., Kianmehr, M. H. and Khani, S. (2008) Mathematical Modeling of Carrot Thin Layer Drying using New Model. Energy Conversion and Management, 49, 201212

[35] Midilli, A., Kucuk, H. and Yapar, Z. A. (2002) New Model for Single Layer Drying. Drying Technology, 20, 1503-1513.

[36] Kumar, N., Sarkar, B. C. and Sharma, H. K. (2012) Mathematical Modeling of the Thin Layer of Hot Air Drying Carrot Pomace. Journal of Food Science and Technology, 49, 33-41.
[37] Demir, V., Gunhan, T. and Yagcioglu, A. K. (2007) Mathematical Modeling of Convection Drying of Green Table Olives. Biosystems Engineering, 98, 47-53.

[38] Gan, P. L. and Poh, P. E. (2014). Investigation on the effect of shapes on the drying kinetics and sensory evaluation study of dried Jackfruit. International Journal of Science and Engineering 7: 193-198.

[39] Afolabi, T. J., Tunde-Akintunde, T. Y. and Adeyanju, J. A. (2015) Mathematical Modelling of Kinetics of Untreated and Pre-Treated Cocoyam Slices. Journal of food sciences Technology, 52, 2731-2740.

[40] Ertekin, C. and Firat, M. Z. (2015). A Comprehensive Review of Thin Layer Drying Models Used in Agricultural Products, Critical Reviews in Food Science and Nutrition, Pg 1 - 73.

[41] Haghi, A. K. (2001) A Mathematical Model of the Drying Process, Acta Polytechnica, 41(3):20 - 23.

[42] Scheffler, G. and Plagge, R. (2005) Drying behavior of building materials. Proceedings of the $7^{\text {th }}$ symposium on building physics in the Nordic countries, Reykjavik, Iceland, $1: 23-30$.

[43] Hii, C. L., Law, C. L. and Cloke, M. (2009). Modeling using a new thin layer drying model and product quality of cocoa. Journal of Food Engineering, 90(2): 191-198. 